\title{
Harmonic holographic microscopy with circularly polarized excitation
}

\author{
Chia-Lung Hsieh*a,b, Rachel Grange ${ }^{\mathrm{a}}$, Ye Pu ${ }^{\mathrm{a}, \mathrm{b}}$, Demetri Psaltis ${ }^{\mathrm{a}, \mathrm{b}}$ \\ ${ }^{a}$ School of Engineering, EPFL, Station 17, 1015 Lausanne, Switzerland; \\ ${ }^{b}$ Department of Electrical Engineering, California Institute of Technology, 1200 East \\ California Boulevard, MC 136-93, Pasadena, California 91125, USA
}

\begin{abstract}
We have developed a luminescent marker using the second harmonic generation (SHG) from noncentrosymmetric $\mathrm{BaTiO}_{3}$ nanocrystals. These nanoparticles are attractive due to their stable, non-saturating and coherent signal with a femtosecond-scale response time and broad flexibility in the choice of excitation wavelength. In this paper, we report the SHG response of $\mathrm{BaTiO}_{3}$ nanocrystals under a circularly polarized excitation. We observed a more uniform SHG signal intensity from nanocrystals of different crystal orientations under a circularly polarized excitation. The threedimensional (3D) SHG fields generated from the SHRIMPs were recorded by a harmonic holographic microscope. Submicron resolution in both lateral and axial directions has been achieved. We show that the circularly polarized excitation is useful for harmonic holographic microscopy.
\end{abstract}

Keywords: second harmonic generation, barium titanate, nanoparticles, digital holography, microscopy, circularly polarized excitation.

\section{INTRODUCTION}

We have developed a luminescent marker using the SHG from noncentrosymmetric nanocrystals which we refer to as "Second Harmonic Radiation IMaging Probes (SHRIMPs)". When a nanocrystal of noncentrosymmetric structure is optically excited at a fundamental frequency, it emits the optical signal at the exact doubled frequency. Only materials with crystalline structures lacking a center of symmetry are capable of efficient SHG. Thus, when imaged at the second harmonic frequency, SHRIMPs provide an effective mechanism of contrast between the markers and the generally unstructured or isotropic environment.

The process of SHG only involves virtual electron energy transition without nonradiative energy loss. Owing to these lossless, virtual transition processes, SHRIMPs emit a stable, non-blinking signal that do not saturate over increasing excitation power. Furthermore, the response time of SHG process is in the femtosecond scale, which allows for the observation of fast dynamic processes over a long time. In addition, SHG is generally a non-resonant process which offers the flexibility in the choice of excitation wavelength. This also results in the flexibility of tuning the wavelength of the SHG signal by changing the excitation wavelength accordingly. The coherent nature of the SHG signal is also a major advantage, providing a possibility to detect the second harmonic signal generated from the nanocrystals with interferometric optical techniques [1-3]. The SHG properties of several kinds of nanocrystals have been recently reported: $\mathrm{BaTiO}_{3}[1,2], \mathrm{ZnO}[4,5], \mathrm{Fe}\left(\mathrm{IO}_{3}\right)_{3}[6], \mathrm{KNbO}_{3}[7], \mathrm{KTiOPO}_{4}(\mathrm{KTP})[3,8]$ and SHG-active crystalline organicinorganic hybrid nanoparticles [9].

The principle of harmonic holography $\left(\mathrm{H}^{2}\right)$ has been demonstrated [1] and its application for cellular imaging has been recently reported [2]. When the digital hologram is recorded at the doubled frequency, it captures both the amplitude and phase information of the SHG object field. Therefore the 3D field distribution, reflecting the 3D distribution of the SHRIMPs could be recorded without scanning. The spatial resolution is limited by the diffraction of light at the half wavelength, and the temporal resolution is limited by the laser pulse duration. Several holographic techniques have been developed for imaging, for example the linear and fluorescent holographic microscopy [10-13]. $\mathrm{H}^{2}$ microscopy is especially suitable for long-term imaging with luminescent markers due to the high imaging contrast provided by detecting the signal at SHG frequency: the background signal (linear background scattering and autofluorescence) can be efficiently removed by optical filters.

*chia-lung.hsieh@epfl.ch; phone +41 21693 7828; fax +41 21693 6930; http://lo.epfl.ch

Advanced Microscopy Techniques, edited by Paul J. Campagnola, Ernst H. K. Stelzer, Gert von Bally, Proc. of SPIE-OSA Biomedical Optics, SPIE Vol. 7367, 73670R · @ 2009 SPIE-OSA · CCC code: 1605-7422/09/\$18 · doi: 10.1117/12.831506 
The polarization dependent SHG response from $\mathrm{BaTiO}_{3}$ nanocrystals has already been experimentally characterized [2]. The crystal orientation determines the polarization dependency of the SHG signal. A theoretical model has also been developed to describe the polarization dependent SHG response of a SHRIMP [2]. The excitation polarization respective to the orientation of the nanocrystal determines the total SHG power radiated from a SHRIMP. Therefore, by measuring the SHG response respective to the excitation polarization, one can find the orientation of the nanocrystal. However, without a complete polarization measurement, the polarization dependent SHG response may complicate the observation. The SHG signal from the nanocrystal can vary in about a factor of 10 due to their orientations. In this paper, we demonstrate a method to mitigate the polarization dependent SHG response of a SHRIMP by exciting it with a circularly polarized light. A more uniform SHG signal from SHRIMPs with different orientations was observed under the circularly polarized excitation. We also successfully imaged these circularly-excited SHRIMPs by a $\mathrm{H}^{2}$ microscope.

\section{SHG RESPONSE OF NANOCRYSTALS}

We used $\mathrm{BaTiO}_{3}$ nanoparticles as SHRIMPs which were commercially obtained from Techpowder. The X-ray diffraction measurement from the suppliers confirmed that the crystal structure is tetragonal, which is non-centrosymmetric, allowing for efficient SHG without any further treatment. Figure 1 is the transmission electron microscope (TEM) picture of the $\mathrm{BaTiO}_{3}$ nanoparticles showing nearly spherical shapes. The size of the nanoparticles varies from $50 \mathrm{~nm}$ to $150 \mathrm{~nm}$.

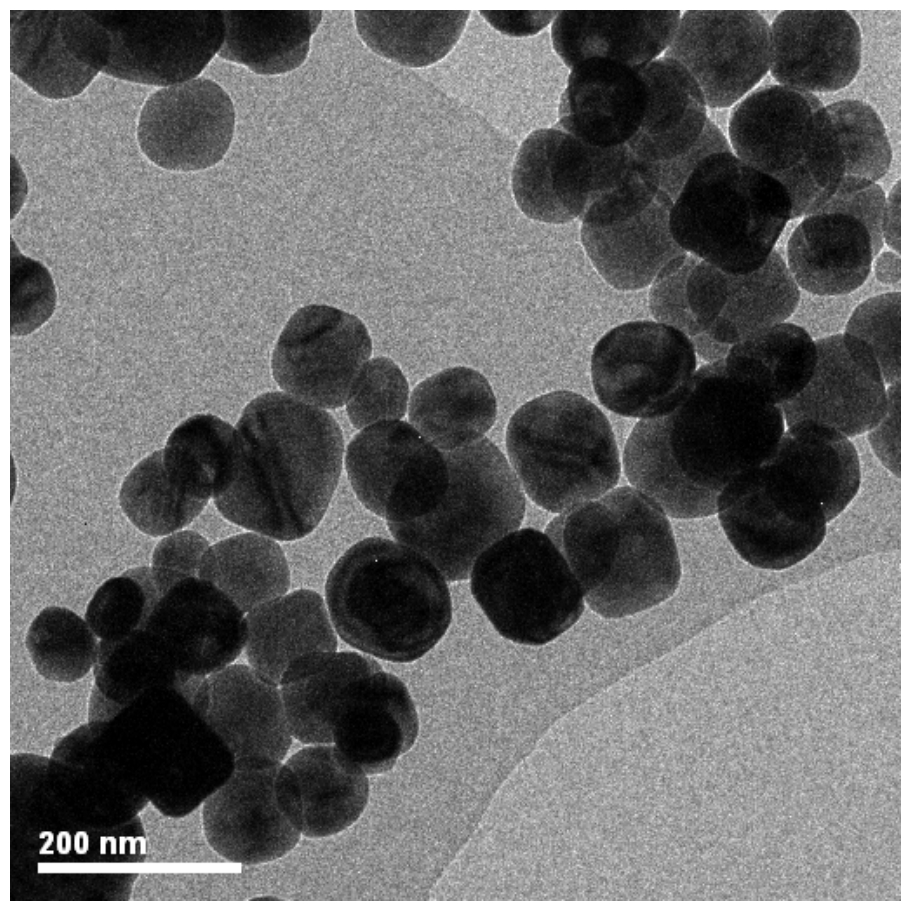

Fig. 1. The TEM picture $\mathrm{BaTiO}_{3}$ nanocrystals. The scale bar is $200 \mathrm{~nm}$

To characterize the SHG response of the SHRIMPs, we deposited the nanoparticles on a glass substrate for imaging. The excitation light source consisted of $150 \mathrm{fs}$ laser pulses centered at $800 \mathrm{~nm}$ wavelength (repetition rate $76 \mathrm{MHz}$ ) from a Ti:sapphire oscillator. The laser beam was slightly focused to reach the peak excitation intensity on the order of $1 \mathrm{GW} / \mathrm{cm}^{2}$ on the sample. The SHG signal was collected by a 1.4 numerical aperture (NA) oil-immersion objective and imaged on an electron multiplying charge coupled device (EMCCD, Andor $\left.\mathrm{iXon}^{\mathrm{EM}_{+}}+885\right)$. We measured the power dependency and the optical spectrum to confirm the SHG signal (Fig. 2). The SHG signal was observed for an hour with less than $5 \%$ decrease under continuous excitation. 


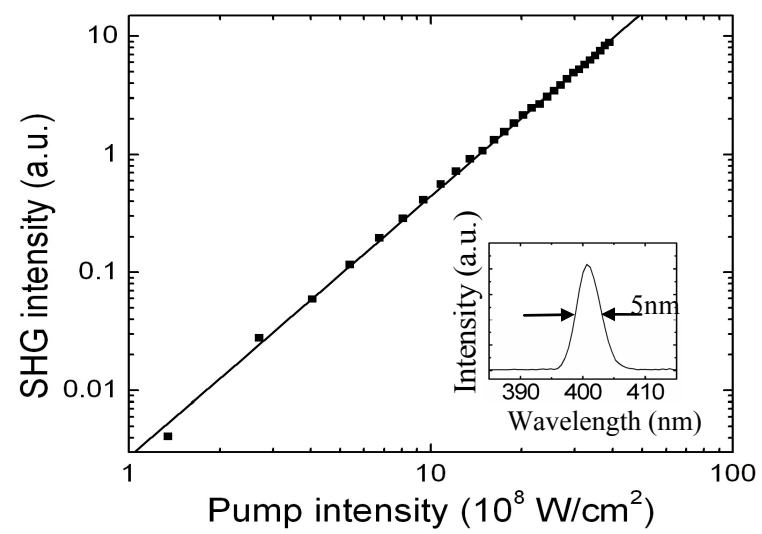

Fig. 2. Power dependence of the $\mathrm{SHG}$ signal from $\mathrm{BaTiO}_{3}$ nanoparticles in double logarithmic scale. The squares are measured results and the solid line is the linear fit with the slope of 2.2. The inset shows the SHG optical spectrum centered at $400.6 \mathrm{~nm}$ with a full width half maximum of $5 \mathrm{~nm}$.

\section{SHG RESPONSE UNDER CIRCULARLY POLARIZED EXCITATION}

The mechanism of SHG from SHRIMPs has been described with a theoretical model in Ref. 2. Briefly, when a SHRIMP is excited by linearly polarized electromagnetic waves at the fundamental frequency, the electric field inside the SHRIMP will induce a nonlinear polarization at the doubled frequency through the second-order optical susceptibilities ( $\chi^{(2)}$ tensor). The magnitude of the nonlinear polarization depends on the nanocrystal orientation relatively to the excitation polarization. The induced nonlinear polarization behaves like a SHG source and radiates the SHG signal. Therefore, the SHG signal from SHRIMPs is polarization dependent. We mitigate the polarization dependent SHG response of SHRIMPs by using circularly polarized excitation. We used the same optical system described in the previous section for the imaging measurement. The sample was $\mathrm{BaTiO}_{3}$ nanocrystals deposited on a glass substrate. We first imaged the sample under linearly polarized excitation. Figure 3 (a) shows the SHG image of the SHRIMPs under linearly polarized excitation. One isolated SHRIMP is clearly shown in Fig. 3 (a). We then imaged the same area with circularly polarized excitation. The circularly polarized excitation is obtained by passing the linearly polarized laser pulses through a quarter-wave plate with proper orientation. Figure 3 (b) shows the SHG image of the SHRIMPs under circularly polarized excitation. In addition to the single SHRIMP which is shown in Fig. 3 (a), two more SHRIMPs appear in Fig. 3 (b). These two SHRIMPs are not visible in Fig. 3 (a) because the SHG response of these two SHRIMPs is weak under that certain linearly polarized excitation. The full width half maximum (FWHM) of the spot sizes in Fig. 3 (a) and (b) are about $200 \mathrm{~nm}$, which is close to the theoretical diffraction limit of our imaging system.
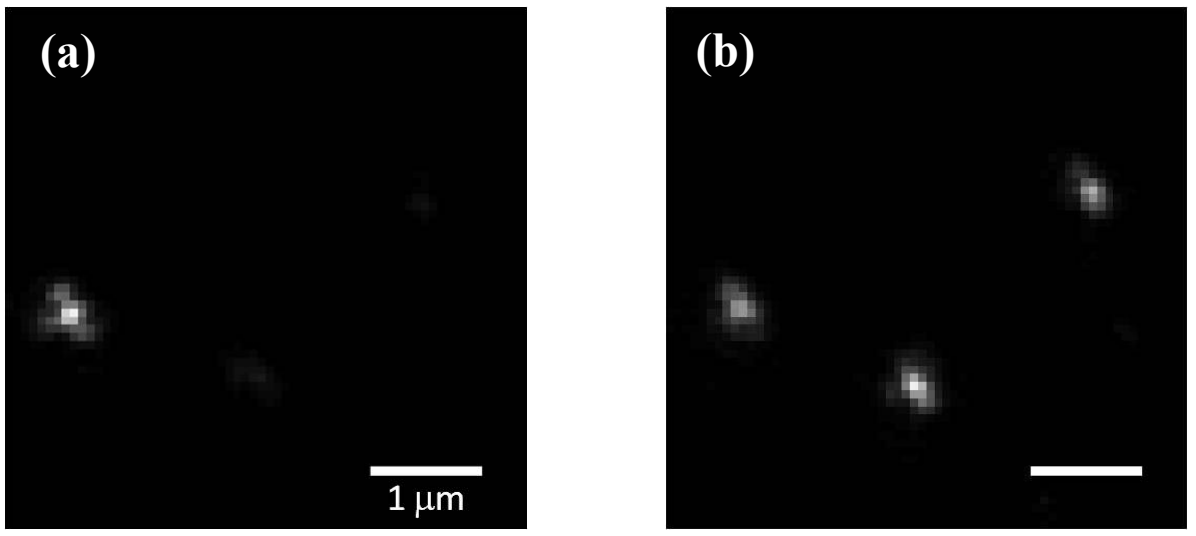

Fig. 3. SHG images of $\mathrm{BaTiO}_{3}$ nanocrystals. (a) Under linearly polarized excitation. (b) Under circularly polarized excitation. The scale bars are $1 \mu \mathrm{m}$. A more uniform SHG signal intensity from the nanocrystals of different orientations is shown in (b). 


\section{H ${ }^{2}$ MICROSCOPY WITH CIRCULARLY POLARIZED EXCITATION}

We have demonstrated the $\mathrm{H}^{2}$ microscopy recently with a linearly polarized excitation $[1,2]$. In this paper, we demonstrate the $\mathrm{H}^{2}$ microscopy with a circularly polarized excitation. The excitation light source is a Ti:sapphire oscillator described previously. The circularly polarized excitation is obtained by passing the linearly polarized laser pulses through a quarter-wave plate with proper orientation. The peak intensity of the excitation is $1.5 \mathrm{GW} / \mathrm{cm}^{2}$. The experimental setup is shown in Fig. 4. The $\mathrm{H}^{2}$ microscope can be understood as a $4 \mathrm{~F}$ imaging system followed by a holographic recording system. The SHG signal from SHRIMPs is collected and optically magnified by a $4 \mathrm{~F}$ system consisting of a 1.4-NA oil-immersion microscope objective and a lens of $20 \mathrm{~cm}$ focal length. We put the EMCCD away from the 4F imaging plane so that the object field could propagate and fill the detection area of EMCCD. The hologram recording distance, i.e. the distance between the SHG image formed by the $4 \mathrm{~F}$ system and the EMCCD, is $20 \mathrm{~cm}$. A plane wave at doubled frequency generated by a separate $\beta$-barium borate (BBO) crystal served as the reference beam. The intensity ratio between the signal maximum and the reference beam is set to be 1:10. The signal and reference pulses were collinearly overlapping both spatially and temporally on the EMCCD, and as a result an on-axis digital hologram was recorded at the SHG wavelength. The inherent twin image has little effect on reconstructed image due to the long hologram recording distance. By recording a digital hologram at the SHG frequency, we capture the 3D SHG field radiated from the SHRIMPs. Through digital reconstruction, we obtain the SHG field at any plane in the sample.

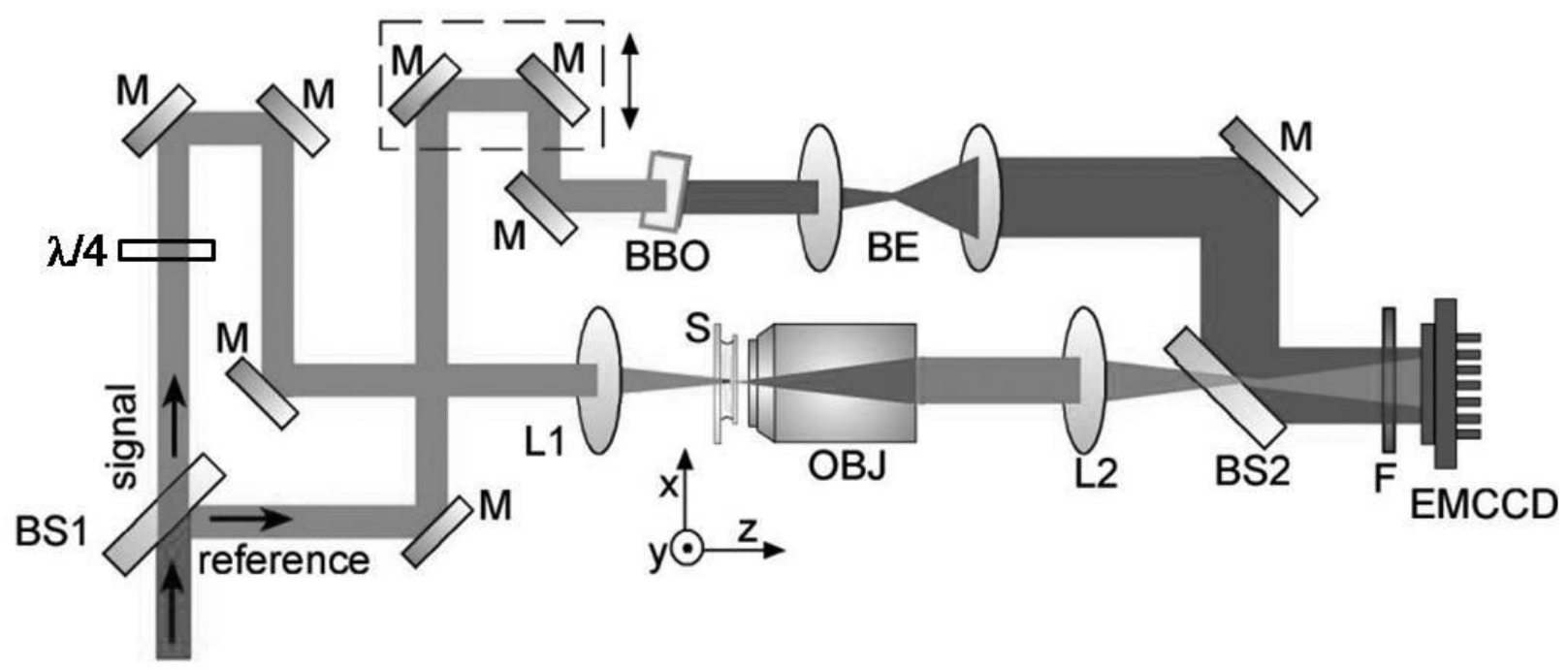

Fig. 4. $\mathrm{H}^{2}$ microscope setup. BS1 and BS2, beam splitters; M, mirror; $\lambda / 4$, quarter-wave plate; L1 and L2, lens; $S$, sample; OBJ, microscope objective; BE, beam expander; F, band-pass filter centered at $400 \mathrm{~nm}$. BS1 splits the laser into signal and reference beams. In the signal arm, L1 slightly focuses the excitation beam into the sample with SHRIMPs. OBJ and L2 form a 4F imaging system to collect and optically magnify the SHG image of SHRIMP. The EMCCD is placed away from the $4 \mathrm{~F}$ imaging plane. A band-pass filter is placed in front of the EMCCD to remove the excitation from the SHG signal. The reference beam goes through a translation stage and a BBO crystal so that the coherent reference SHG laser pulses are generated and can be temporally and spatially overlapped with the signal on the EMCCD. The signal and reference beams are combined collinearly by BS2 and therefore an on-axis digital hologram is recorded on the EMCCD.

We imaged the same SHRIMPs shown in Fig. 3 by the $\mathrm{H}^{2}$ microscope. In the first measurement, the SHRIMPs were excited by a linearly polarized light. The corresponding $\mathrm{H}^{2}$ reconstructed image is shown in Fig. 5 (a) where only one SHRIMP is visible, which matches with the conventional SHG image (Fig. 3 (a)). In the next measurement, the SHRIMPs were excited by a circularly polarized light. The corresponding $\mathrm{H}^{2}$ reconstructed image is shown in Fig. 5 (b) where three SHRIMPs appear as the conventional SHG image (Fig. 3 (b)). It is worth noting that in both measurements, 
we used a linearly polarized reference beam. Therefore, the $\mathrm{H}^{2}$ reconstructed images do not reflect the SHG signal from SHRIMPs of all the polarizations.
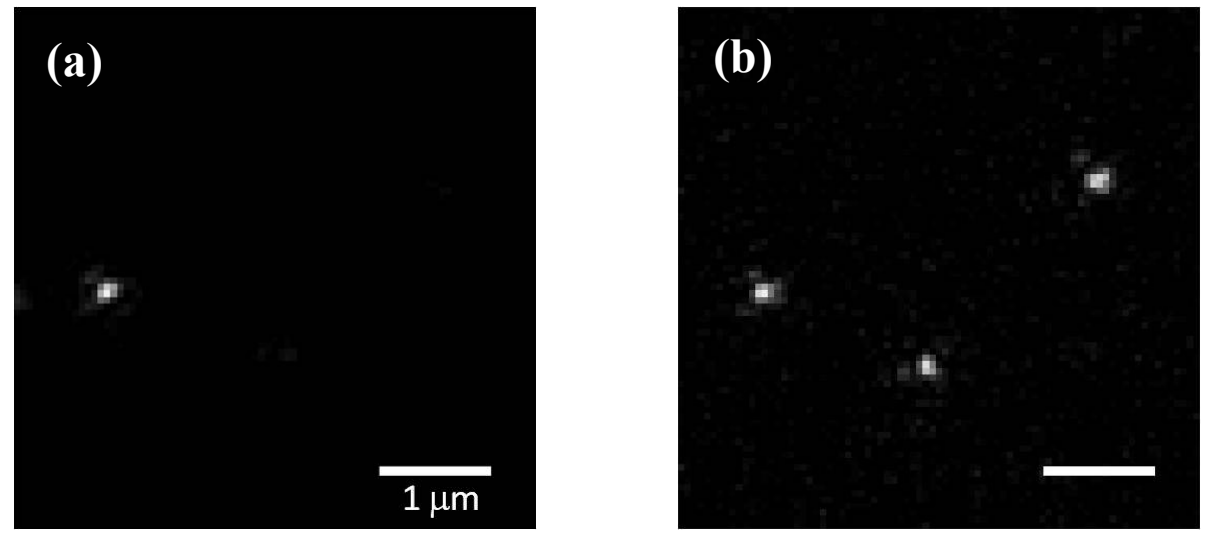

Fig. 5. The $\mathrm{H}^{2}$ reconstructed images of the same SHRIMPs shown in Fig. 3. (a) Under linearly polarized excitation. (b) Under circularly polarized excitation. The scale bars are $1 \mu \mathrm{m}$.

By digitally reconstructing the SHG field at different planes from the recorded hologram, we could obtain a 3D distribution of SHG field in the sample without scanning. We did a 3D reconstruction for the middle SHRIMP shown in Fig. 5 (b), and the result is shown in Fig. 6. The lateral and axial resolutions are about $200 \mathrm{~nm}$ and $400 \mathrm{~nm}$ respectively which are the diffraction limit at the SHG wavelength of our imaging system.
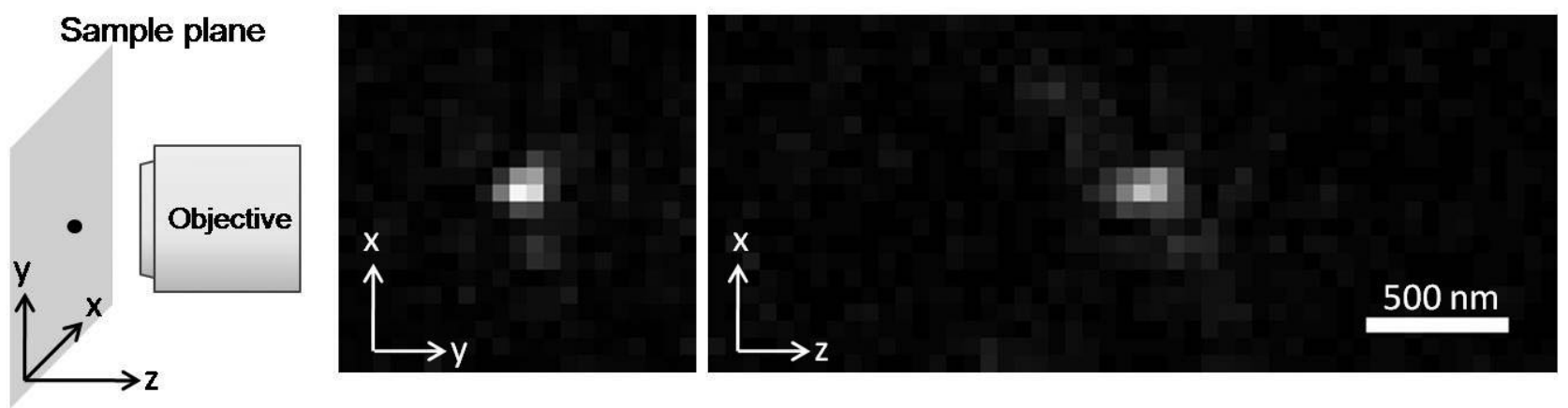

Fig. 6. The $\mathrm{H}^{2}$ reconstructed section and cross-section images of one isolated SHRIMP. Sub-micron resolution has been achieved both in lateral and axial directions.

\section{CONCLUSION}

We demonstrated the use of $\mathrm{BaTiO}_{3}$ nanoparticles as SHRIMPs for SHG imaging. We examined the $\mathrm{SHG}$ response from SHRIMPs under linearly and circularly polarized excitation. The SHRIMPs of different orientations show a more uniform SHG signal under a circularly polarized excitation. We also imaged the SHRIMPs under circularly polarized excitation with a $\mathrm{H}^{2}$ microscope. About 200-nm lateral resolution and 400-nm axial resolution of the $\mathrm{H}^{2}$ microscope have been achieved. 


\section{REFERENCES}

[1] Pu, Y., Centurion, M., and Psaltis, D., "Harmonic holography: a new holographic principle," Appl. Opt. 47, A103A110 (2008).

[2] Hsieh, C.-L., Grange, R., Pu Y., Psaltis, D., "Three-dimensional harmonic holographic microscopy using nanoparticles as probes for cell imaging," Opt. Express 17, 2880-2891 (2009).

[3] Xuan, L. L., Brasselet, S., Treussart, F., Roch, J. F., Marquier, F., Chauvat, D., Perruchas, S., Tard, C., and Gacoin, T., "Balanced homodyne detection of second-harmonic generation from isolated subwavelength emitters," Appl. Phys. Lett. 89, 121118 (2006).

[4] Kachynski, A. V., Kuzmin, A. N., Nyk, M., Roy, I., and Prasad, P. N., "Zinc oxide nanocrystals for nonresonant nonlinear optical microscopy in biology and medicine," J. Phys. Chem. C 112, 10721-10724 (2008).

[5] Johnson, J. C., Yan, H. Q., Schaller, R. D., Petersen, P. B., Yang, P. D., and Saykally, R. J., "Near-field imaging of nonlinear optical mixing in single zinc oxide nanowires," Nano Letters 2, 279-283 (2002).

[6] Bonacina, L., Mugnier, Y., Courvoisier, F., Le Dantec, R., Extermann, J., Lambert, Y., Boutou, V., Galez, C., and Wolf, J. P., "Polar Fe(IO3)(3) nanocrystals as local probes for nonlinear microscopy," Appl. Phys. B-Lasers and Optics 87, 399-403 (2007).

[7] Nakayama, Y., Pauzauskie, P. J., Radenovic, A., Onorato, R. M., Saykally, R. J., Liphardt, J., and Yang, P. D., "Tunable nanowire nonlinear optical probe," Nature 447, 1098-1101 (2007).

[8] Sandeau, N., Le Xuan, L., Chauvat, D., Zhou, C., Roch, J. F., and Brasselet, S., "Defocused imaging of second harmonic generation from a single nanocrystal," Opt. Express 15, 16051-16060 (2007).

[9] Delahaye, E., Tancrez, N., Yi, T., Ledoux, I., Zyss, J., Brasselet, S., and Clement, R., "Second harmonic generation from individual hybrid MnPS3-based nanoparticles investigated by nonlinear microscopy," Chem. Phys. Lett. 429, 533-537 (2006).

[10] Marquet, P., Rappaz, B., Magistretti, P. J., Cuche, E., Emery, Y., Colomb, T., and Depeursinge, C., "Digital holographic microscopy: a noninvasive contrast imaging technique allowing quantitative visualization of living cells with subwavelength axial accuracy," Opt. Lett. 30, 468-470 (2005).

[11] Kemper, B., Carl, D., Schnekenburger, J., Bredebusch, I., Schaefer, M., Domschke, W., von Bally, G., "Investigation of living pancreas tumor cells by digital holographic microscopy," J. Biomed. Opt. 11, 034005 (2006).

[12] Schilling, B. W., Poon, T. C., Indebetouw, G., Storrie, B., Shinoda, K., Suzuki, Y., and Wu, M. H., "Threedimensional holographic fluorescence microscopy," Opt. Lett. 22, 1506-1508 (1997).

[13] Rosen, J., and Brooker, G., "Non-scanning motionless fluorescence three-dimensional holographic microscopy," Nat. Photonics 2, 190-195 (2008). 